\title{
Context Revisited: A brief survey of research in context aware multimedia systems
}

\author{
Thanos Demiris \\ European Dynamics S.A. \\ 209 Kifissias Ave. \& Arkadou str. \\ 15124 Maroussi, Athens, GREECE \\ +302108094500 \\ A.M.Demiris@Computer.org
}

\begin{abstract}
The definition of context is context-depended itself... Context information is processed and has been used for making information delivery and presentation more efficient. The use of context is gaining in importance with the increasing number of pervasive computing applications. The efficiency of information delivery to mobile devices, which still suffer from limited resources (e.g. processing, display and power) and communications bandwidth, determines the acceptability of services by the users; even more so in cases of demanding multimedia information delivery, which takes up bandwidth and processing power. For multimedia applications and services to become widely accepted the correct context may play a significant role. In this brief survey and introductory paper we try to identify the characteristics of recent research effort in context-aware systems, mainly in multimedia applications. We initially define context and subsequently classify context determination techniques.
\end{abstract}

\section{Keywords}

Context, context-aware multimedia, personalization.

\section{INTRODUCTION}

As with many other developments in computing, context-aware and ubiquitous computing systems were prototyped for the first time in Xerox's Palo Alto Research Labs in the framework of the PARCTAB project in the late 80ies, early 90ies. The introduction of small portable devices (mainly used for the display of information by centralized systems deploying infrared information exchange) triggered the thought of computing outside the limits of the desktop computer. Mark Weiser first introduced the term ubiquitous computing and reported about it in [1], while Shilit et.al. reflected thoughts on context-awareness for the first time in [2].

The definition of context it context-depended itself... Definitions

Permission to make digital or hard copies of all or part of this work for personal or classroom use is granted without fee provided that copies are not made or distributed for profit or commercial advantage and that copies bear this notice and the full citation on the first page. To copy otherwise, or republish, to post on servers or to redistribute to lists, requires prior specific permission and/or a fee.

MOBIMEDIA 2007, August 27-29, Nafpaktos, Greece

Copyright $\odot 2007$ ICST 978-963-06-2670-5

DOI 10.4108/ICST.MOBIMEDIA2007.1861 existing thus far address context by means of examples or synonyms, which heavily rely on the experience and the background of the research teams addressing context. Hence in some cases context is related to the computing and communication resources, while other works focus more on higher level concepts dealing with the user state. Both approaches are legitimately called context-aware, as shown in the next section.

Context-aware computing is dealing mainly with the two principle aspects, namely 1.) capturing context elements and combining them (deducing the actual context) as well as 2.) matching the estimated context with the information to be delivered, thus selecting the most appropriate information out of a much larger superset. In this paper we will firstly present a survey of the three different ways of capturing context information and subsequently present ongoing research in context-aware computing and applications as well as future research directions and priorities in the realm.

Context-awareness research is motivated by the general perception of the research community that the combination of several context values may lead to a better understanding of the overall context of a user [8] and assist the information filtering process, thus leading to targeted information delivery, more suitable for the time-span of attention of a human user.

\section{USER CONTEXT}

In this paper we will refer mainly to the user context and much less to the usage or operational context, which may be useful in robotic applications or networking and communications optimization. We will examine definitions and work mainly related to increasing the information value for a user by focusing on information that is more relevant in a given situation, i.e. a superset of what is known as personalization. The type of information that will be examined is multimedia information.

The context contains information about the user him-/herself (preferences, habits, mood, physiological condition) as well as information about the environment (location, viewing field, physical conditions such as light, noise, humidity etc., co-location with others, interactions, group dynamics). Something that is not taken into account often is the state of the computing system itself (load, other applications executing, time since last power-up etc.), which may deliver some information about the tasks of the user on the one hand, and help shape up context estimation in terms of resources, level of detail etc. (if the computing device is 
overloaded with high priority tasks, the exact determination of the context may not be useful and a coarse context estimation may be more adequate).

Dey and Abowd introduced a generic definition of context in [1] where they define it as "any information that can be used to characterize the situation of an entity. An entity is a person, place, or object that is considered relevant to the interaction between a user and an application, including the user and applications themselves". The authors differentiate between primary and secondary context. Location, identity, time, and activity are the primary context types for characterizing the situation of a particular entity. Primary context are all types of contextual information about an entity that may be used to determine additional information about that entity, i.e. the secondary context information. In the terminology of the authors primary context may "index" secondary context.

A recent work by Yingyi et.al. [5] is dealing with the quality of context, trying to identify sources of problems for inaccurate context estimations, that may lead applications to fail or deliver inadequate information. They identify also the sequence of steps for context estimation, which consists in:

- Data acquisition

- Data interpretation and representation as context

- Context aggregation

- Adaptation inference

- Context reasoning

A different approach, but with the same target of decreasing the effects of ill calculated context is introduced by Ranganathan et.al. in [6], where the context sensing entities deliver information characterized by uncertainty. The authors introduce a framework for treating context information by means of probabilistic logic, fuzzy logic and Bayesian networks. Their contribution is also very interesting in terms of modeling context, and their classification of

- context providers,

- context synthesizers and

- $\quad$ context consumers

very much corresponds to the layered concept of context underlying the present paper. In addition, they identify context provider lookup services, aiming at detecting the most appropriate sensors in the vicinity and history lookup services, where past context estimations may be retrieved and reused for present decisions.

\section{THE ESTIMATION OF CONTEXT}

The user context may be captured and processed in numerous ways. Initial work used interfaces to sensors to determine context by itself, but since the end of the nineties it is very common to deal with context in dedicated subsystems that deliver this information to multiple applications. The determination of context takes thus place on multiple layers, starting with the collection of data, continuing with the initial processing and fusion, subsequently reasoning about the actual context based on the elementary information collected and finally taking actions based on this information. Baldauf et.al. present in [10] a layered conceptual framework, which starts with the collection of values from different sensors and reaches up to the application as follows:

- Sensors

- Raw Data Retrieval

- $\quad$ Preprocessing

- Storage and management of context information

- Context-aware Application

In their paper about location management in pervasive systems [13], Indulska and Sutton introduced 2003 a classification of sensors that is generally applicable to context sensors (not just location). They differentiate between:

- Physical sensors: this category basically summarizes all hardware sensors that capture values of the environment or the users themselves, such as position, motion, light, temperature etc.

- Virtual sensors: mainly contextual information collected from the interaction of the user with software systems, e.g. a the mouse motion determining that the user is actually sitting at the PC, a calendar entry suggesting that the user may be away from home etc.

- Logical sensors: higher level context estimation through the arbitrary combinations of physical and virtual sensors.

In the following a novel perspective of context estimation classification is introduced, based on the involvement of the user. It is merely a different viewing angle to the information presented by other colleagues in the work referenced thus far.

\subsection{Interactive determination}

The "easiest" way to determine the user's context, from a developer's point of view, is to ask for it... Hence many systems, especially the ones aiming at personalization, chose the "intrusive path" prompting the user to explicitly input information about their gender, age, interests etc. As this has proven in many cases inefficient and rejected by many users as too "intrusive" or "time consuming", the tendency is to try to estimate user context by less explicit methods. A typical example is related to music. There the user is given the opportunity to annotate their music stored with additional labels related to their preferences, such as "star" rating and mood. The selection of a song characterized by a mood description of the user makes it a lot easier for the system to determine a list of related songs addressing a similar mood. This context estimation could be characterized as "context estimation by example".

\subsection{Sensorial Estimation}

There are multiple kinds of sensors that collect measurements from the users and their environments:

- Ambient sensors: are mostly fixed sensors that monitor environmental parameters. Typical examples include temperature sensors, humidity, light etc.

- Wearable sensors: are positioned on the clothes or body of the user and measure activity or physiological parameters. 
- Transdermal sensors: are minimally invasive sensors that are applied in form of patches and take measurements of biophysiological parameters.

- Implantable sensors: a new category of sensors that measures biophysiological values very accuratel and continuously. The biocompatibility of these sensors is still an issue in many cases. Such sensors are aiming mainly at cases of chronically ill patients. Along with the transdermal sensors they are not expected to find wide use in applications other than healthcare.

Cameras are a very promising but also annoying means of determining user context. Using a camera, a system may be able to detect many parameters of the user's context, but the user will constantly feel observed and his or her privacy severely violated. Cameras may be used to determine the mood of a know face, by applying face detection technology. The very basic matching of the user's mood to the multimedia content available may be based on a set of selection criteria starting with explicit annotations (metadata) and going all the way up to intelligent mood detectors, e.g. as the one described by Dimitrova in [4] related to color.

An important aspect in context estimation is the time dimension. It is extremely important to be able to synchronize the clocks of various sensors and the reading of various information sources in order to be able to deduce the correct user context.

\subsection{Analytical determination of context: long- term and historical context}

The user context has a very strong time dependency. Apart from the current context of the user, an overall user context may be of value for the correct delivery of information. The context of use of an application by a given user provides the means for determining many user characteristics by analyzing previous encounters of the user with the system. Data mining technology may be deployed to detect recurrent patterns in a context repository or system logfiles.

This category is widely used in multimedia systems, with the most typical example being the personalized EPGs (electronic programming guides) for interactive television. In this context the selections of the user are collected and analyzed over time to create the best possible recommendations for broadcasted or video-on-demand content.

\subsection{Models of context}

The work presented here coincides with the summarization of context models presented by Baldauf et.al. in [10] and the work by Strang and Linnhoff-Popien [15]. According to this classification the modeling of context may take place by the following means:

- Key-value pairs: the simplest method of describing contextual information is to enlist the various attributes to be measured and store their values in key-value pairs.

- Markup models: typical representation by means of $\mathrm{RDF} / \mathrm{S}$ etc. Markups are used in some of the following representations as well, but simple markup models mentioned here are static in nature and simply describe in a somehow more structured way the information captured also by key-value models.
- Graphical models: semi-formal models, such as the Unified Modeling Language (UML) may be used for the description of context. The advantage of such models is that they are considered more intuitive and are easier to integrate with UML models of the rest of the applications.

- Object-oriented models: The concepts of encapsulation and inheritance are used to model basic context types that may be extended in future applications of a model. For simple context models object-oriented models may be somehow adding some overhead.

- Logic based models: formal description of context, which allows for high-level reasoning. The subsequent ontological modeling seems more adequate, since it contains a certain degree of formalism, allows for inferencing and is easier to handle than rule-based models.

- Ontological models: the modeling addresses contextual concept determination and allows for defining relationships between different concepts. Reasoning may be applied to these concepts. Ontologies are widely spread due to their linkage with the semantic web and make out a "trend" in current modeling approaches.

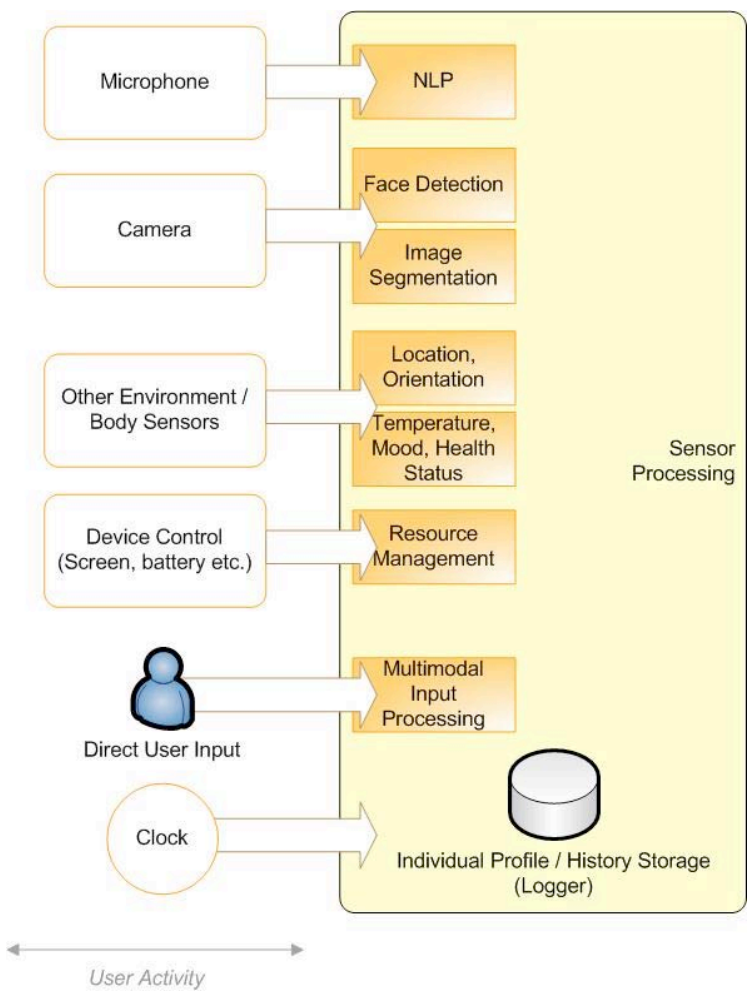

Figure 1 A variety of different information sources may be used to determine user context. 


\section{PAST, PRESENT AND FUTURE OF CONTEXT AWARE MULTIMEDIA APPLICATIONS}

In a very nicely structured presentation in [8], some indicative initial applications of context-aware systems are presented. These applications were targeting a set of services related mainly to location detection neglecting most of the additional context information. Hence the applications were targeting tourism and cultural heritage [12], smart calendars and notification systems as well as targeted advertisement systems in shopping malls etc. delivering information mainly based on the position of users in relation to a display or important item in their vicinity. In recent years research started targeting the home environment, aiming at supporting applications for the smart-home environment with contextual information. This research started shifting the focus also towards higher level context, such as mood, since this type of information may play a role in home entertainment and automation. A survey of context-aware applications for the smart home environment may be found in [13].

Current context-aware multimedia applications mainly focus on determining the location of the user in order to deliver the most appropriate multimedia presentation, be it a video-clip, a commercial or a multimedia presentation related to an archaeological site of interest. In addition, the device characteristics may be used to adapt the bandwidth of the multimedia files streamed to the end-user, but this is work much closer to communications design. The personalization of content delivery in the case of video-on-demand and IPTV definitely serve as the basis for advancing research in context estimation. One reason for determining the context of the user during an IPTV session, is in order to enrich the actual multimedia streaming with additional relevant information that is appropriate for the given users at the given time. A typical example of this work may be found in [16].

A more complex case is related to a new type of entertainment, called pervasive gaming, as presented by Linner et.al. in [17]. Pervasive gaming is the new type of computer-based entertainment, where the action leaves the computer screen in a room to enter a much larger environment, such as the complete house or even the metropolitan settings. Pervasive games combine multiple aspects, such as social interaction etc. An essential characteristic of all games of this type is that they heavily rely on location information to be carried out. The work presented by Linner is a typical multimedia application that deploys a centralized context service management component and models context by means of description logic to accomplish efficient management of events and delivery of multimedia information. Pervasive gaming is interesting not only in the context of contextawareness... but also in terms of networking, since demanding multimedia information often needs to be exchanged among participants. This leads to many peer-to-peer solutions.

Applications that have been traditionally monitor-bound are now also following the general trend and become more and more device independent. An application area investigated earlier than others is healthcare. There various concepts exist, where applications of context-aware systems aim at reducing the workflow in clinical settings, as discussed by the author in [18], or Upkar Varshney in [19]. Healthcare applications deploy vast amounts of multimedia information in order to deliver efficient information to healthcare personnel, but also as information services to increase patient awareness. Services to help patients prepare for an examination etc. started deploying multimedia means (mainly video) to deliver the information necessary in a manner most appropriate for the viewer under consideration (not too complicated on the one hand, not too boring on the other).

Current trends go basically in two directions. One is related to the augmented information visualization, where the environment of the user becomes enriched with information visualized by various means. Context-aware applications were thus far deploying only one device to visualize information. Currently the research is aiming at systems that address multiple device deployment, e.g. in a smart-home or smart-office environment, where the user is moving from one place to the next without carrying a portable computer in many cases. A seamless handover of information is necessary for the users to harvest advantages of context-aware applications. A typical example is the display of multimedia on a TV screen in a living room and then the continuation of this playback either on a portable device, when one of the users is leaving the home environment of the activation of an additional display in another room, once another user leaves the living room to go there.

The other research direction is targeting the basic functionality of context-aware systems and addresses the problems of security and privacy. The latter may play a significant role in the acceptance of many technological developments in this area, since people start feeling increasingly observed in their everyday activities and might reject new technologies and applications if they feel their privacy is threatened.

\section{CONCLUSION}

The present work is a brief survey of research related to contextaware systems in general as well as in relation to multimedia. There are many ways to classify research in context-aware systems. In this work we presented the processing steps necessary to determine context, as well as the different types of context determination, namely sensorial, interactive and analytical. A discussion of various context-aware applications deploying multimedia information indicates that a constantly increasing number of application domains relies on these technologies. A critical aspect in the future will be research related to privacy protection, in order for the technologies developed to become widely accepted.

\section{REFERENCES}

[1] M. Weiser, "Some Computer Science Problems in Ubiquitous Computing," Communications of the ACM 36, No. 7, 74-83, July 1993

[2] Bill N. Schilit, Norman Adams, and Roy Want, "Contextaware computing applications", Proc. Workshop on Mobile Computing Systems and Applications, Pages 85-90, Santa Cruz, CA, December 1994

[3] Anind K. Dey and Gregory D. Abowd: "Towards a Better Understanding of Context and Context-Awareness", Proc. 1st International Symposium on Handheld and Ubiquitous Computing (HUC '99), June 1999

[4] Nevenka Dimitrova: "Context and Memory in Multimedia Content Analysis”, IEEE Multimedia, pp.7-11, July 2004

[5] Yingyi Bu, Tao Gu, Xianping Tao, Jun Li, Shaxun Chen, Jian Lu, "Managing Quality of Context in Pervasive 
Computing," qsic, pp. 193-200, Sixth International Conference on Quality Software (QSIC'06), 2006

[6] Anand Ranganathan, Jalal Al-Muhtadi, Roy H. Campbell, "Reasoning about Uncertain Contexts in Pervasive Computing Environments" IEEE Pervasive Computing, Vol. 3, Nr. 2, pp. 62-70, April 2004

[7] Pengkai Pan, Carly Kastner, David Crow, Glorianna Davenport. "M-Studio: an Authoring Application for Context-aware Multimedia." ACM Multimedia 2002, Juanles-Pins, France, 2002

[8] Guanling Chen and David Kotz "A Survey of Context-Aware Mobile Computing Research", Technical Report TR2000381, Dartmouth College, 2000

[9] Andy Harter, Andy Hopper, Pete Steggles, Andy Ward and Paul Webster, "The Anatomy of a Context-Aware Application”, Wireless Networks, Nr. 8, pp.187-197, 2002

[10] Matthias Baldauf, Schahram Dustdar and Florian Rosenberg, "A survey on context-aware systems", Int. J. Ad Hoc and Ubiquitous Computing, Vol. 2, No. 4, 2007

[11] Spiridoula Koukia, Maria Rigou, Spiros Sirmakessis “The Role of Context in m-Commerce and the Personalization Dimension", IEEE/WIC/ACM, WI-IAT 2006 Workshops, pp. 267-276, 2006

[12] Keith Cheverst, Nigel Davies, Keith Mitchell and Adrian Friday, "Experiences of Developing and Deploying a Context-Aware Tourist Guide: The GUIDE Project", Proc. 6th annual international conference on Mobile computing and networking, pp. 20-31, 2000
[13] Jadwiga Indulska, Peter Sutton, "Location management in pervasive systems”, Proc. CRPITS '03 Australasian information security workshop conference on ACSW frontiers, pp.143-151, 2003

[14] Sven Meyer, and Andry Rakotonirainy, "A survey of Research on Context-Aware Homes", Proc. CRPITS '03 Australasian information security workshop conference on ACSW frontiers, pp. 159-168, 2003

[15] Strang, T., Linnhoff-Popien, C.: “A context modeling survey" Proc. UbiComp 1st International Workshop on Advanced Context Modelling, Reasoning and Management, Nottingham, pp.34-41, 2004

[16] Amit Thawani, Srividya Gopalan and Sridhar V. "Context Aware Personalized Ad Insertion in an Interactive TV Environment ", TV'04: the 4th Workshop on Personalization in Future TV - Methods, Technologies, Applications for Personalized TV, 2004

[17] David Linner, Fabian Kirsch, Ilja Radusch, Stephan Steglich "Context-aware Multimedia Provisioning for Pervasive Games", Proc. $7^{\text {th }}$ IEEE International Symposium on Multimedia (ISM'05), pp. 60-68, December 2005

[18] Demiris, A.M., Ioannidis, N. "Context Awareness and Nomadic Devices featuring Advanced Information Visualization in Clinical Routine", Journal of Telecommunications and Information Technology, Nr. 4, pp. 121-128, 2005

[19] Upkar Varshney, "Pervasive Healthcare", IEEE Computer, vol. 36, no. 12, pp. 138-140, Dec., 2003 